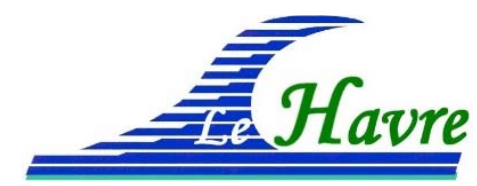

XVIèmes Journées Nationales Génie Côtier - Génie Civil
Le Havre, 2020

DOI:10.5150/jngcgc.2020.065 (C) Editions Paralia CFL

disponible en ligne - http://www.paralia.fr - available online

\title{
Restauration écologique en milieu estuarien : quels outils pour quels objectifs?
}

\section{Cécile CAPDERREY ${ }^{1}$, Maud BERLINCOURT ${ }^{2}$, Maïa AKOPIAN ${ }^{3}$}

1. BRGM, Direction des Risques et de la Prévention (DRP), Unité Risques Côtiers et Changement Climatique (R3C), 3 Av. Claude Guillemin, BP 36009, Orléans, France. c.capderrey@brgm.fr

2. GIP Seine-Aval, Hangar C, Espace des Marégraphes, 76176 Rouen, France. mission.inter-estuaires@ofb.gouv.fr

3. Office Français de la Biodiversité, 5, square Félix Nadar, 94300 Vincennes, France. maia.akopian@ofb.gouv.fr

\section{Résumé :}

Les estuaires sont des zones de transition entre les eaux douces et marines aux caractéristiques hydrologiques morphologiques et sédimentaires très variables dans le temps et dans l'espace. Ils font partie des systèmes les plus anthropisés au monde pour lesquels il est devenu plus qu'urgent d'agir concrètement en réhabilitant durablement leurs fonctions écologiques et de fait, les services écosystémiques qu'ils assurent pour la protection et la survie des populations humaines. A l'échelle de la façade Manche Atlantique, les activités de restauration écologique menées sur la dernière décennie ont souvent fait état de nombreux échecs. Face à ce constat, l'Agence Française pour la Biodiversité (devenue Office Français de la Biodiversité en 2020) finance depuis 2015 un travail sur la construction d'un cadre méthodologique pensé pour les difficultés de la restauration écologique en estuaire. Outre la capitalisation des retours d'expérience nécessaires à l'impulsion des démarches, ce travail a également permis d'inventorier les modèles mathématiques mobilisables pour définir des objectifs de restauration. Leur utilisation a été discutée à la lumière des différentes problématiques écologiques rencontrées, des types de fonctionnements d'un estuaire (hydro-morpho-sédimentaire, biogéochimique, trophique et comme support au cycle de vie des espèces) et enfin, a été mise en perspective avec le niveau de connaissances scientifiques actuel et les impératifs d'action, pour penser et déployer efficacement la restauration écologique.

\section{Mots clés :}

Estuaires, Restauration écologique, Fonctions écologiques, Services écosystémiques, Modèles mathématiques, Objectifs de restauration.

\section{Introduction}

Les estuaires subissent depuis longtemps des modifications de leur structure physique et chimique (endiguement, urbanisation, pollutions, navigation, aquaculture, etc.). Ces modifications altèrent leur fonctionnement et réduisent leur capacité à procurer de 


\section{Thème 6 - Gestion durable des zones littorales et estuariennes}

nombreux services écosystémiques (zones tampons face aux évènements climatiques, zones de rétention et de recyclage des nutriments et polluants, perte des habitats essentiels au cycle de vie d'organismes marins et estuariens (TEMMERMAN et al., 2013, COSTANZA et al., 2014). Les récents consortiums internationaux (IPBES, IPCC) et cadres législatifs imposés par les réglementations nationales et internationales (DCE, DCSMM, DHFF, DO, etc.) pointent plus que jamais l'urgence d'agir au niveau des zones littorales, et particulièrement estuariennes, où de nombreux enjeux socio-écologiques sont menacés à moyen terme par l'élévation du niveau de la mer, l'augmentation de la fréquence d'événements extrêmes, la compression côtière, etc. Il est donc essentiel de maintenir et de restaurer le fonctionnement écologique des estuaires et de fait les services écosystémiques qu'ils procurent. Toutefois, la restauration écologique pose de nombreux défis atteignant parfois les limites du savoir écologique actuel. Outre le manque de connaissances écologiques encore important sur le fonctionnement des estuaires et la difficulté d'acquérir des données dans un environnement fortement impacté, les spécificités de ces milieux rendent les actions compliquées. Les estuaires sont naturellement complexes en termes de composantes, de dynamiques et d'interactions (marée, gradients de température, d'oxygène, de salinité, etc.) où il est difficile de séparer les effets d'origine naturelle de ceux d'origine anthropiques (ELLIOTT \& QUINTINO, 2007). Dans ce contexte, la restauration de fonctions écologiques s'annonce très complexe à appréhender sans connaissance écologique pointue des réponses des écosystèmes (état initial, temps de réponse des écosystèmes : hystérésis, rôle des habitats estuariens etc.). Le besoin d'agir est urgent et le contexte actuel de changement global impose d'agir avec les connaissances disponibles et celles en cours d'acquisition. S'appuyer sur les retours d'expérience et employer des outils solides est d'autant plus important pour définir les objectifs de restauration, mesurer leur atteinte et gagner en efficacité. Ce travail a donc pour objectif(1) d'établir un état des lieux des modèles mobilisés au regard des différents types de dysfonctionnements estuariens ; (2) de discuter de leur modalités d'application (niveau de données, de connaissances, de compétences, etc.) afin de considérer leur emploi pour répondre aux problématiques rencontrées à l'échelle des estuaires français.

\section{Démarche}

2.1 Le lien entre les habitats, les processus et les fonctions écologiques

Les activités de restauration visent idéalement à rétablir un lien entre la structure de l'habitat et la réalisation des fonctions écologiques (figure 1). Le chemin généralement adopté, consiste donc à identifier et à modifier les variables de l'habitat (les structures abiotiques et biotiques), afin d'améliorer ou d'optimiser la réalisation des processus s'y déroulant (ensemble des réactions, modifications biologiques, physiques et chimiques 


\section{XVİ̀mes Journées Nationales Génie Côtier - Génie Civil \\ Le Havre 2020}

mises en jeu dans les transferts de matière et d'énergie) pour in fine améliorer ou optimiser l'efficacité des fonctions écologiques.

La mesure de l'efficacité des fonctions réalisées permet alors de renseigner sur l'efficacité des procédures de restauration (c'est-à-dire que les variables structurantes de l'habitat ont été correctement identifiées et modifiées par les actions de restauration).

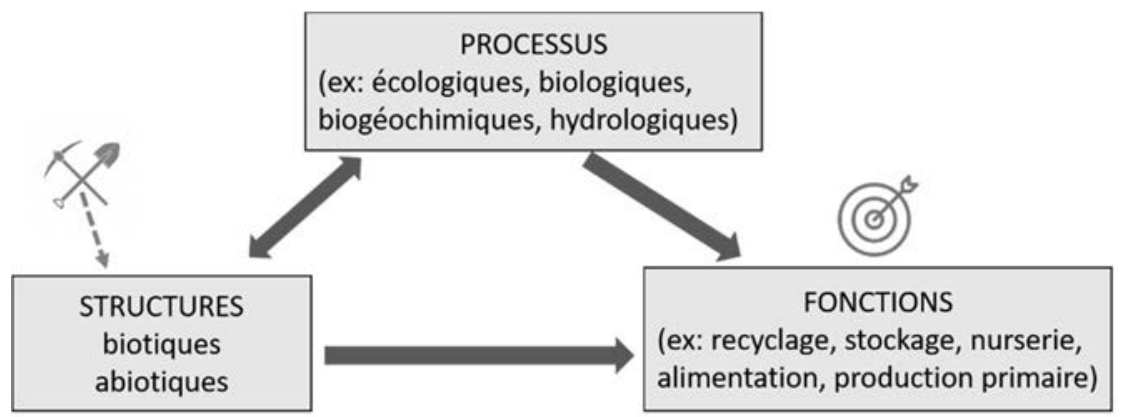

Figure 1. Schéma des liens entre structures des habitats, processus s'y déroulant et fonctions écologiques associés.

\subsection{Des outils pour les objectifs de restauration}

Définir des objectifs de restauration requiert d'identifier clairement les entités sur lesquelles l'action doit être portée pour obtenir une récupération effective de la fonction ciblée. L'objectif de la restauration doit donc être si possible, quantifiable et mesurable. Les modèles sont donc des outils importants pour comprendre des processus complexes, tester des hypothèses, prédire le comportement des écosystèmes et répondre à des questions du type "que se passe-t-il si ?". Le recensement des modèles a été établi à partir d'une analyse bibliographique reposant sur 250 articles scientifiques.

\section{Mise à plat des modèles autour des grands types de fonctionnement estuarien et des problématiques associées}

Compte tenu de la forte dynamique des estuaires, leur fonctionnement complexe s'appréhende spatialement (du micro-habitat à la mosaïque d'habitats estuariens) et temporellement (cycle des marées, phénomènes saisonniers dans les apports de matière, etc.). Ainsi 4 perspectives de fonctionnement totalement liées entre-elles, couvrent cette dynamique spatio-temporelle (figure 2). 


\section{Thème 6 - Gestion durable des zones littorales et estuariennes}

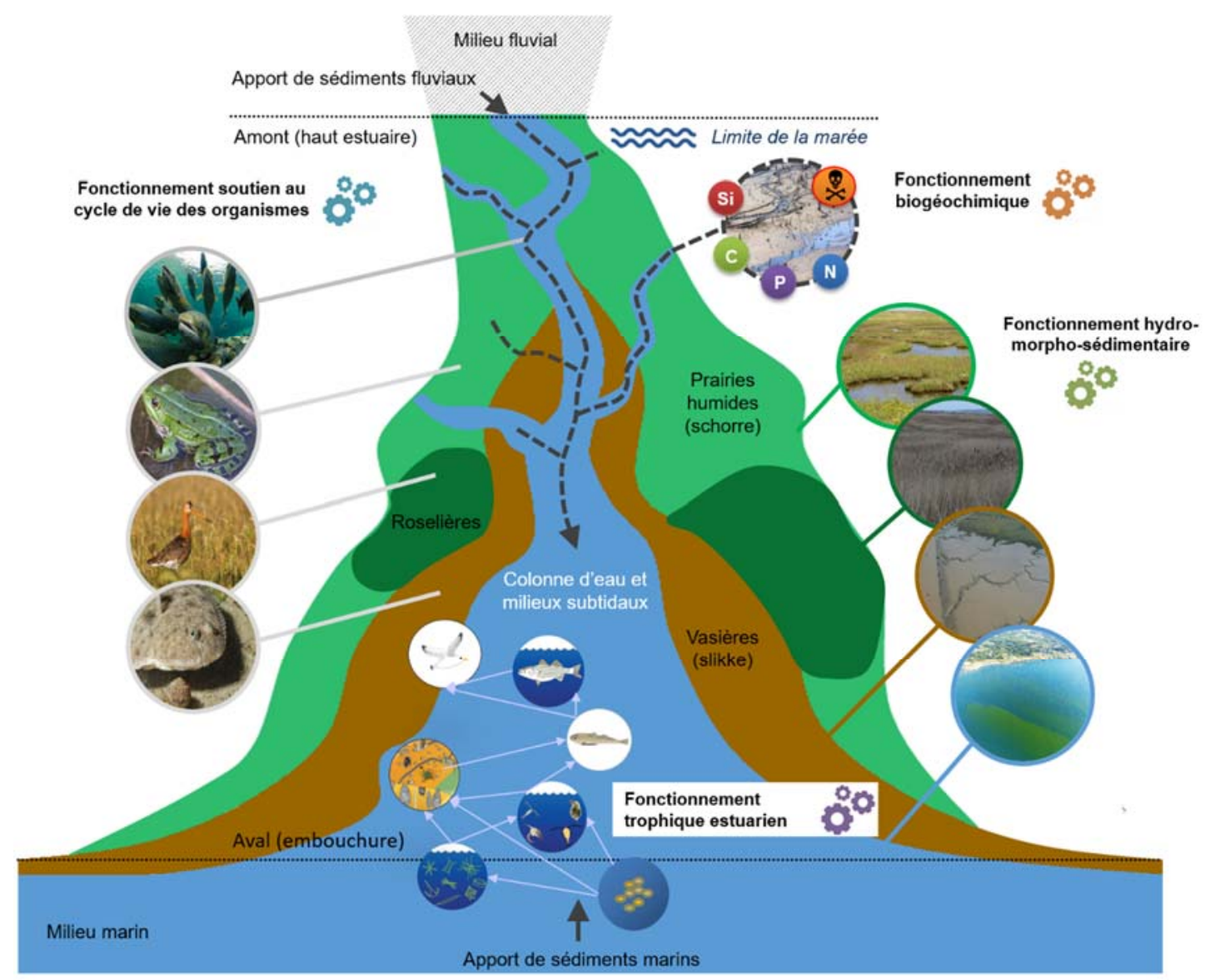

Figure 2. Différentes perspectives du fonctionnement estuarien.

\subsection{Le fonctionnement biogéochimique}

Situés en "bout de chaîne", les estuaires sont des catalyseurs biogéochimiques qui reçoivent fortes quantités de nutriments et de polluants. Le fonctionnement biogéochimique englobe la dynamique des processus permettant la transformation de la matière et de l'énergie, voire des contaminants. Les perturbations dans les flux de transport de nutriments modifient fortement le déroulement des processus, c'est pourquoi l'évaluation quantitative des flux est un défi. Les modèles biogéochimiques aident à répondre aux problématiques d'eutrophisation, d'hypoxie, de chute de production biologique et de contamination. Ils aident à identifier les sources responsables et informent numériquement sur la quantité de nutriments à diminuer et sur la réponse biologique à cette diminution. Développés pour de nombreux estuaires de tailles différentes (ex: Guadiana, Chesapeake Bay, Yangtze, Gironde, etc.), les modèles biogéochimiques sont utilisés comme outil de surveillance et d'aide à la décision pour les politiques de gestion de l'eau. Ils reproduisent les cycles biogéochimiques de l'écosystème et nécessitent pour cela de prendre en compte les différents forçages qui s'appliquent. C'est pourquoi ils sont souvent couplés avec un modèle dynamique pour avoir une représentation réaliste de la dynamique des éléments. Par exemple, selon les 


\section{XVI'̀mes Journées Nationales Génie Côtier - Génie Civil \\ Le Havre 2020}

environnements et les processus biogéochimiques qui s'y déroulent, les modèles biogéochimiques peuvent être couplés (1) avec un modèle de transport sédimentaire (ex : CUGIER \& LE HIR, 2002), (2) avec un modèle de transport réactionnel (ex : VOLTA et al., 2014), ou encore (3) à différents compartiments biologiques (ex : ECOMARS-3D, MENESGUEN \& DUSSAUZE, 2014). Depuis leur émergence il y a une trentaine d'années, les modèles biogéochimiques intègrent de plus en plus de processus et sont très complexes. Ils ont permis d'apporter des réponses solides et numériquement appuyées aux problématiques écologiques entrainées par l'eutrophisation et l'hypoxie. Leur utilisation reste déterminée par une grande quantité de données et de solides compétences mathématiques (procédures de calibration et de validation des modèles). L'attente de simplification et de banalisation de l'emploi de ces modèles dans un futur proche ne doit cependant pas décourager l'acquisition de données de qualité sur les estuaires les moins documentés ni la mise en place de méthodologies pour améliorer cette acquisition.

\subsection{Le fonctionnement hydro-morpho-sédimentaire}

Déclinable sur une échelle allant du mètre à celle de l'estuaire, il regroupe les aspects dynamiques reliés au transport des sédiments et à la dynamique des structures spatiales et granulométriques sédimentaires créées par les patrons de transport. Parmi ces structures, les zones intertidales sont le résultat de nombreux processus hydrodynamiques, sédimentaires et biologiques (période et fréquence d'inondation, géomorphologie, caractéristiques des sols, interactions végétation-sédiments, bioturbation). Les enjeux de restauration sont multiples, à la fois d'un point de vue fonctionnel (production de matériel organique et recyclage/déstockage des nutriments) que structurel (par exemple, perte de la diversité des communautés végétales jouant sur les processus d'accrétion). Les outils déployés dans la littérature pour répondre à la large gamme des problématiques écologiques associées à la perte de fonctionnalité des zones intertidales sont très variés. Aussi bien représentés par des modèles empiriques que physiques, ils permettent :

- à une échelle locale :

de modéliser le couplage entre la biomasse végétale et la sédimentation (ex: FAGHERAZZI et al., 2012); de modéliser la formation et la dynamique des chenaux intertidaux (ex: TEMMERMAN et al., 2019; D'ALPAOS et al., 2007; VANDENBRUWAENE et al., 2012) et de prédire les relations entre les surfaces à restaurer et les dimensions des chenaux (ex : HOOD et al., 2015); voire de modéliser les impacts des communautés benthiques sur la dynamique sédimentaire (ex : LUMBORG et al., 2006 ; ORVAIN et al., 2017);

- à l'échelle de l'estuaire :

de prédire l'évolution et la pérennité des zones intertidales face au changement climatique (ex: ZHANG \& GORELICK, 2014) ou encore de modéliser les réponses hydrodynamiques à grande échelle (ex : KHANGAONKAR \& YANG, 2011). 


\section{Thème 6 - Gestion durable des zones littorales et estuariennes}

Les modèles identifiés permettent tous d'appréhender la dynamique du transport sédimentaire à différentes échelles mais ils considèrent encore les processus écologiques à un niveau très simplifié. Le couplage entre la géomorphologie et l'écologie est certes souvent quantifié mais souvent approché par des équations décrivant les processus physiques. Ces approches sont récentes et leur application à des problématiques de restauration est encore partielle. Les auteurs reconnaissent néanmoins régulièrement le besoin pressant de recherche pour évaluer plus précisément les influences réciproques de la biologie (végétale et animale) sur la morphologie et le transport sédimentaire, les processus d'érosion et de dépôts liés aux sédiments cohésifs, la sédimentologie de la matière organique et les effets de la granulométrie et de la microtopographie sur l'érosion et le dépôt. Cela n'empêche pour autant pas leur utilisation, à condition de mettre l'accent sur l'acquisition de données comme les taux de sédimentation ce qui est aujourd'hui possible grâce à l'amélioration des techniques d'acquisition (ex : suivis altimétriques).

\subsection{Le fonctionnement soutien au cycle de vie des organismes}

La mosaïque des habitats estuariens soutient le cycle de vie de très nombreux taxons. Face à la perte et à la dégradation des habitats essentiels à leur cycle de vie, les problématiques écologiques sont nombreuses et diverses selon les espèces considérées et leur stade de vie. Les questionnements s'inscrivent ainsi sur tout un gradient d'actions (augmentation de la surface des habitats, amélioration de la qualité des habitats résiduels, amélioration de la qualité globale de l'eau, amélioration, rétablissement de la connectivité entre les habitats existants et leur accessibilité). Les outils identifiés permettent d'établir des relations entre la réponse biologique des espèces et les facteurs environnementaux. Ils sont employés pour simuler des améliorations dans les effectifs, cibler des espèces candidates à la restauration et proposer des leviers dont on sait qu'ils entraineront une bonne réponse biologique. Ils regroupent :

Des approches statistiques "dites statiques" relativement classiques dans leurs approches comme les modèles linéaires généralisés, modèles additifs généralisés, modèles boostés généralisés, "random forests", etc..., (ex : LE PAPE et al., 2014 ; RADINGER et al., 2015 ; TEICHERT et al., 2016). Ces approches sont accessibles (logiciels d'analyse classique avec comme principal élément déterminant, la qualité de la donnée densité abondance), simples à comprendre et à expliquer à condition de consacrer du temps à l'interprétation des sorties de modèles, mais fournissent en revanche peu d'information sur la capacité d'accueil des habitats. La plupart des modèles identifiés peuvent être couplés à un SIG et devenir des interfaces utiles pour la gestion. Des modèles plus dynamiques et complexes intègrent la connectivité du paysage et les forçages environnementaux. Ils permettent par exemple, de prendre en compte les aspects spatial et temporel de la marée dans l'accessibilité des habitats par les taxons mobiles (ex : poissons) (ex : LE PICHON \& ALP., 2018) ou permettent de modéliser la dynamique de dispersion de l'ichtyoplancton (ex : LETT et al., 2008). Des modèles vont également 


\section{XVI'èmes Journées Nationales Génie Côtier - Génie Civil \\ Le Havre 2020}

jusqu'à modéliser l'évolution des réponses physiologiques et comportementales individuelles face à des modifications environnementales (ex : STILLMAN et al., 2016). Ces modèles permettent d'aller au-delà de "l'habitat favorable" en prenant en compte la complexité des cycles de vie et des patrons de dispersion de certaines espèces, processus clé dans la structuration et la dynamique des communautés. Leur emploi reste conditionné par l'acquisition de données à haute résolution spatiale et la mobilisation des compétences techniques nécessaires. En ciblant précisément les variables environnementales qui déterminent en grande partie les réponses biologiques, l'ensemble des modèles identifiés couvre un vaste champ de questionnement autour des fonctions écologiques (alimentation, reproduction, refuge, nourricerie, etc.). Les principaux freins à l'utilisation de l'ensemble des modèles présentés résident davantage dans les manques de connaissances écologiques sur les liens habitat-espèce, et la qualité/quantité des données, que dans les compétences techniques pour faire tourner les modèles.

\subsection{Le fonctionnement trophique}

Au sein des écosystèmes, les espèces sont organisées en réseaux trophiques. Il s'agit de l'ensemble des interactions entre différents niveaux d'alimentation qui sont reliés par des flux d'énergie et de matière. Cette organisation intègre en tant que descripteurs de nombreux aspects de la biodiversité, parmi lesquels la productivité de l'écosystème, sa stabilité ou sa résilience. L'extinction de multiples espèces impliquées dans le fonctionnement a notamment pour effet de diminuer la productivité des écosystèmes en modifiant les transferts d'énergie et leur efficacité. Les activités de restauration visent à rétablir des conditions favorables pour des espèces cibles, mais oublient trop souvent que ces espèces sont des composantes d'un réseau trophique auquel elles contribuent plus ou moins activement dans l'espace et dans le temps. Initialement développés pour évaluer les effets de la pêche sur les écosystèmes, les modèles trophiques ont été étendus et modifiés pour inclure des effets d'origine anthropique comme l'eutrophisation ou les modifications physiques de l'habitat, ce qui en fait donc des outils prometteurs pour aider à la restauration (ex : ECOPATH, CHRISTENSEN \& WALTERS 2004). Ces modèles offrent un cadre de travail holistique (l'intégralité des écosystèmes) et fonctionnel (capable de quantifier des flux) mais aussi couplable avec de nombreuses problématiques écologiques (scénarios d'eutrophisation, de modification de salinité, d'augmentations ou de diminutions de surface physique d'habitat). Bien que la construction des modèles requiert un paramétrage complexe et des jeux de données extensifs reposant sur la description précise des relations au sein de l'écosystème concerné, ils occupent une part de plus en plus importante dans la gestion des ressources marines et bénéficient d'une réceptivité croissante sur le milieu estuarien, à mesure que la compréhension de la complexité des réseaux progresse et que les appels à les considérer dans la restauration écologique sont de plus en plus forts. 


\section{Thème 6 - Gestion durable des zones littorales et estuariennes}

\section{Conclusion}

Dans un contexte de pressions physiques croissantes et de changement climatique, l'urgence de restaurer les fonctions des estuaires est un défi qui laisse de moins en moins de place aux démarches empiriques; bien que ce soit encore souvent le seul moyen dont on dispose.

Les modèles sont des outils puissants pour comprendre et prédire l'efficacité des actions de restauration. Ils permettent de formaliser de façon quantitative le savoir acquis ou requis pour explorer différents scénarios écologiques voire économiques afin d'appuyer les politiques environnementales. Ils sont aujourd'hui de plus en plus mobilisés dans les projets de restauration et pour la plupart d'entre eux, la banalisation de leur utilisation pourrait en faire des outils dont il deviendrait difficile de se passer dans un futur proche. Sur la base de cette analyse, un cadre de réflexion a visée opérationnelle a été proposé (figure 3). Il propose une orientation vers des outils pour aider à penser les objectifs de restauration de façon plus quantifiée et ainsi de maximiser la probabilité de les atteindre.

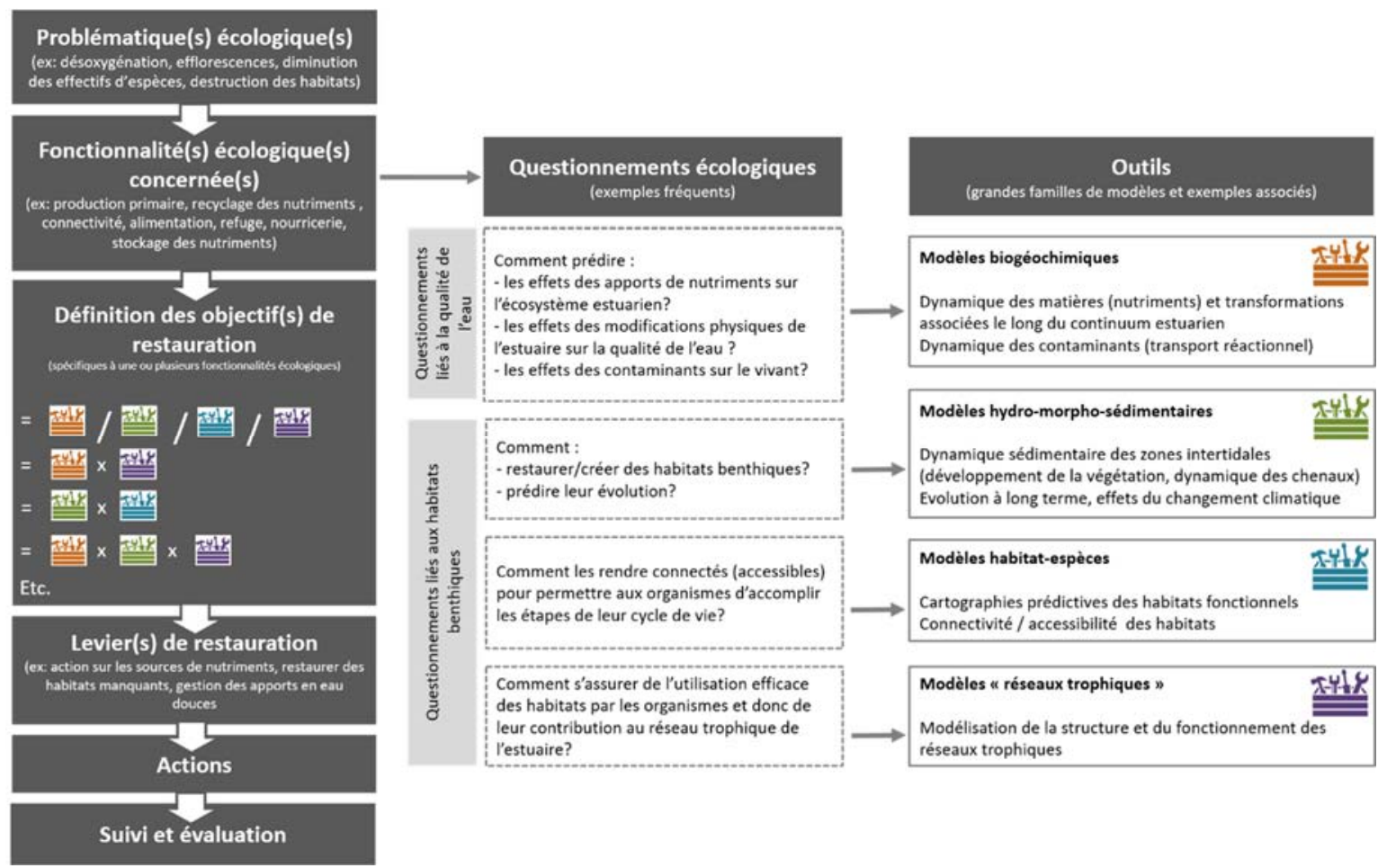

Figure 3. Synthèse de la démarche vers l'emploi des modèles pouvant contribuer à une définition quantifiée des objectifs de restauration.

\section{Références bibliographiques}

COSTANZA R., DE GROOT R., SUTTON P., VAN DER PLOEG S., ANDERSON S.J., KUBISZEWSKI I., FARBER S., TURNER R.K. (2014). Changes in the global value of ecosystem services. Global environmental change. 26: 152-158. 


\section{XVİ̀mes Journées Nationales Génie Côtier - Génie Civil \\ Le Havre 2020}

CHRISTENSEN V., WALTERS C.J. (2004). Ecopath with Ecosim: methods, capabilities and limitations. Ecological modelling, Vol. 172(2-4), pp 109-139. https://doi.org/10.1016/j.ecolmodel.2003.09.003

CUGIER P., LE HIR P. (2002). Development of a 3D hydrodynamic model for coastal ecosystem modelling. Application to the plume of the Seine River (France). Estuarine, Coastal and Shelf Science, Vol. 55, pp 673-695. https://doi.org/10.1006/ecss.2001.0875

D’ALPAOS A., LANZONI S., MARANI M., BONOMETTO A., CECCONI G., RINALDO A. (2007). Spontaneous tidal network formation within a constructed salt marsh: Observations and morphodynamic modelling. Geomorphology, Vol. 91, pp 186-197. https://doi.org/10.1016/j.geomorph.2007.04.013

ELLIOTT M., QUINTINO V. (2007). The estuarine quality paradox, environmental homeostasis and the difficulty of detecting anthropogenic stress in naturally stressed areas. Marine Pollution Bulletin, Vol. 54(6), pp 640-645. https://doi.org/10.1016/j.marpolbul.2007.02.003

FAGHERAZZI S., KIRWAN M.L., MUDD S.M., GUNTENSPERGEN G.R., TEMMERMAN S., D'ALPAOS A. VEN DE KOPPEL J., RYBCZYK J.M., REYES E., CRAFT C., CLOUGH J. (2012). Numerical models of salt marsh evolution: Ecological, geomorphic, and climatic factors. Reviews of Geophysics, Vol. 50(1). https://doi.org/10.1029/2011RG000359

HOOD W.G. (2015). Predicting the number, orientation and spacing of dike breaches for tidal marsh restoration. Ecological Engineering, Vol. 83, pp 319-327. https://doi.org/10.1016/j.ecoleng.2015.07.002

KHANGAONKAR T., YANG Z. (2011). A High-Resolution Hydrodynamic Model of Puget Sound to Support Nearshore Restoration Feasibility Analysis and Design. Ecological Restoration, Vol. 29 (1-2), pp 173-184. https://doi.org/10.3368/er.29.1-2.173 LE PAPE O., DELAVENNE J., VAZ S. (2014). Quantitative mapping of fish habitat: a useful tool to design spatialised management measures and marine protected area with fishery objectives. Ocean \& coastal management, Vol. 87, pp 8-19. https://doi.org/10.1016/j.ocecoaman.2013.10.018

LE PICHON C., ALP M. (2018). ANACONDHA ; ANAlyse spatiale de la CONnectivité Des HAbitats fonctionnels pour les poissons à l'échelle de l'estuaire. Projet de recherche Seine-Aval 5.

LETT C., VERLEY P., MULLON C., PARADA C., BROCHIER T., PENVEN P., BLANKE B. (2008). A Lagrangian tool for modelling ichthyoplankton dynamics. Environmental Modelling \& Software, Vol. 23(9), pp 1210-1214. https://doi.org/10.1016/j.envsoft.2008.02.005

LUMBORG U., ANDERSEN T.J., PEJRUP M., (2006). Modelling the effect of macrozoobenthos and microphytobenthos on cohesive sediment transport on an intertidal mudflat. Estuarine, Coastal and Shelf Science, Vol. 68(1-2), pp 208-220. https://doi.org/10.1016/j.ecss.2005.11.039 


\section{Thème 6 - Gestion durable des zones littorales et estuariennes}

MENESGUEN A., DUSSAUZE M. (2014). Détermination des "bassins récepteurs" marins des principaux fleuves français de la façade Manche-Atlantique, et de leurs rôles respectifs dans l'eutrophisation des masses d'eau DCE et des sous-régions DCSMM.

ORVAIN F. (2017). Programme de recherche Seine-Aval 5 : BARBES (Associations biologiques en relation avec le transport sédimentaire : développement d'un modèle de bioturbation par les ingénieurs d'écosystèmes en estuaire de Seine RADINGER J., WOLTER C., KAIL J. (2015). Spatial scaling of environmental variables improves species-habitat models of fishes in a small, sand-bed lowland river. PloS one, Vol. 10(11), e0142813. https://doi.org/10.1371/journal.pone.0142813

STAGG C.L., MENDELSSOHN I.A. (2011). Controls on resilience and stability in a sediment subsidized salt marsh. Ecological Applications, Vol. 21(5), pp 1731-1744. https://doi.org/10.1890/09-2128.1

STILLMAN R.A., WOOD K. A., GOSS-CUSTARD J.D. (2016). Deriving simple predictions from complex models to support environmental decision-making. Ecological modelling, Vol. 326, pp 134-141. https://doi.org/10.1016/j.ecolmodel.2015.04.014

TEICHERT N., BORJA A., CHUST G., URIARTE A., LEPAGE M. (2016). Restoring fish ecological quality in estuaries: implication of interactive and cumulative effects among anthropogenic stressors. Science of the Total Environment, Vol. 542, pp 383-393. https://doi.org/10.1016/j.scitotenv.2015.10.068

TEMMERMAN S., MEIRE P., BOUMA T.J., HERMAN P.M., YSEBAERT T., DE VRIEND H.J. (2013). Ecosystem-based coastal defence in the face of global change. Nature, Vol. 504(7478), pp 79-83. https://doi.org/10.1038/nature12859

TEMMERMAN S., VAN BELZEN J., GOURGUE O., SCHWARZ C., BOUMA T.J., VAN DE KOPPEL J. (2019). A bio-geomorphic model for smart design of climate resilient tidal marsh restoration. In Geophysical Research Abstracts (Vol.1)

VOLTA C., ARNDT S., SAVENIJE H.H., LARUELLE G.G., REGNIER P. (2014). CGEM (v 1.0): a new, cost-efficient biogeochemical model for estuaries and its application to a funnel-shaped system. Geoscientific Model Development, Vol. 7, pp 1271-1295. https://doi.org/10.5194/gmd-7-1271-2014

VANDENBRUWAENE W., MEIRE P. TEMMERMAN S. (2012). Formation and evolution of a tidal channel network within a constructed tidal marsh. Geomorphology, Vol. 151, pp 114-125. https://doi.org/10.1016/j.geomorph.2012.01.022

ZHANG H., GORELICK S. (2014). Coupled impacts of sea-level rise and tidal marsh restoration on endangered California clapper rail. Biological Conservation, Vol. 172, pp 89-100. https://doi.org/10.1016/j.biocon.2014.02.016 